\title{
Cancerous pericarditis presenting as cardiac tamponade in a 68-year-old man with pancreatic adenocarcinoma: a case report
}

\author{
Sachie Kiryu', Zensho Ito ${ }^{1 *}$, Masashi Ishikawa', Takafumi Akasu, ${ }^{1,2}$ Yoshihiro Matsumoto', Shinichi Hirooka²,
} Masayuki Saruta ${ }^{3}$ and Shigeo Koido ${ }^{1}$

\begin{abstract}
Introduction: Pericardial effusion is a rare complication of pancreatic cancer. We report a case of cardiac tamponade secondary to pancreatic cancer.

Case presentation: A 68-year-old Japanese man was diagnosed as having pancreatic cancer during surgery and received chemotherapy for 28 months after the diagnosis. He was admitted to the emergency room with severe dyspnea. Echocardiography revealed pericardial effusion with severe hypofunction. Emergency pericardial drainage was performed to maintain hemodynamics, which resulted in the elimination of $450 \mathrm{~mL}$ of blood and the maintenance of circulatory dynamics. Cytological examination of the pericardial fluid revealed atypical cells and tumor cells suggesting adenocarcinoma.

Conclusions: To our knowledge, pancreatic cancer complicated with cancerous pericarditis has not been previously documented. This case highlights the extreme severity of pericardial effusion, a sign of progressive disease, secondary to pancreatic cancer. In the case of neoplastic pericardial effusion, an extremely poor prognosis must be considered.
\end{abstract}

Keywords: Pancreatic cancer, Cardiac tamponade

\section{Introduction}

Malignant pericardial effusion caused by carcinomatous pericarditis is a complication of advanced malignancy. Pericardial effusion may cause cardiac tamponade and sudden death if not properly controlled. Cardiac tamponade as a complication of pancreatic cancer is very rare. Cardiac tamponade caused by carcinomatous pericarditis induces the retention of pericardial fluid, causing pericardial effusion, and must be treated promptly as an oncologic emergency [1]. Emergency treatment is necessary to prevent sudden death and relieve symptoms of

\footnotetext{
* Correspondence: zensho@jikei.ac.jp

'Division of Gastroenterology and Hepatology, Department of Internal Medicine, The Jikei University School of Medicine, Kashiwa Hospital, 163-1 Kashiwa-shita, Kashiwa, Chiba 277-8567, Japan

Full list of author information is available at the end of the article
}

pericardial effusion in patients with neoplastic cardiac tamponade [2]. To the best of our knowledge, pericardial involvement in patients with pancreatic cancer has never been reported. Here, we report a case of metastatic pancreatic cancer complicated by pericardial effusion.

\section{Case report}

A 68-year-old Japanese man was suspected of having pancreatic cancer 3 years previously due to an increase in carbohydrate antigen (CA)19-9. Abdominal computed tomography (CT) revealed a 26-mm hypoechoic mass on the head of his pancreas and liver metastasis. Endoscopic ultrasonography (EUS) and fine-needle aspiration (FNA) of the pancreatic mass revealed adenocarcinoma. He was diagnosed as having Stage IV unresectable 
pancreatic cancer, and gemcitabine (GEM) plus nanoparticle albumin-bound paclitaxel (nab-PTX) doublet chemotherapy was administered. After 2 years of GEM/ nab-PTX chemotherapy, progression of the disease was confirmed; therefore, the chemotherapy regimen was changed to GEM + tegafur-gimeracil-oteracil (S1). Six months later, he experienced dyspnea and visited the clinic after a few days of experiencing symptoms. CT revealed pericardial effusion and bilateral pleural effusion. Laboratory tests revealed elevated C-reactive protein $(\mathrm{CRP})$ at $6.23 \mathrm{mg} / \mathrm{dL}$. Pneumonia was treated with betalactam antibiotics. Therefore, he was diagnosed as having pneumonia and heart failure. The following day, his blood oxygen saturation was $90 \%$ on room air, and he had tachycardia (110 beats per minute) and hyperthermia $\left(37.2^{\circ} \mathrm{C}\right)$. His blood pressure was $86 / 67 \mathrm{mmHg}$. Respiratory and circulatory failure was managed in an emergency room. Electrocardiography revealed sinus tachycardia, and echocardiography revealed a severe pericardial swinging heart motion (Fig. 1a and b). Pericardial drainage was performed through a pericardial window, resulting in the drainage of $450 \mathrm{~mL}$ of blood, and the pericardial fluid was subjected to cytological examination. Cytological examination of the fluid revealed tumor cells indicating adenocarcinoma. Blood levels of CA19-9 and carcinoembryonic antigen (CEA) were $5.8 \mathrm{ng} / \mathrm{mL}$ and $50 \mathrm{IU} / \mathrm{mL}$, respectively, which gradually increased during the course of treatment, suggesting pancreatic cancer progression. A pericardial drainage tube was removed after improvement in his general condition. One week after the surgical procedure, intravenously administered chemotherapy with GEM/nab-PTX was re-initiated. Sixteen days after re-initiation of chemotherapy, he was admitted to the emergency room and died of cardiac arrest due to severe respiratory failure without evidence of recurrent pericardial effusion.

In this case, a pathological autopsy was performed. On macroscopic examination, the parietal pericardium and visceral pericardium had adhered, indicating cancerous pericarditis (Fig. 2a). Pericardial effusion was not observed. On microscopic examination, a large number of tumor cells had infiltrated the pericardium, and some had infiltrated the lymph vessels of the myocardium (Fig. 2b and c). These results suggest pericardial metastasis of pancreatic cancer.

\section{Discussion}

Carcinomatous pericarditis is observed in $10-20 \%$ of patients with malignancies, with the most common malignancies being lung cancer, breast cancer, leukemia, and malignant lymphoma. According to a study by Berge and Sievers, malignant tumor metastasis involving the pericardium was observed in $50 \%$ of autopsies of patients with metastatic malignant tumors [3]. Pericardial metastasis does not frequently cause cardiac tamponade. However, cardiac tamponade is a life-threatening condition requiring prompt diagnosis and treatment. Cardiac tamponade is the lethal phase of neoplastic pericarditis resulting from cardiac compression and the accumulation of pericardial fluid. Intrapericardial pressure increases and cardiac relaxation is inhibited, leading to low output; patients often experience dyspnea, hypotension, tachycardia, cold sweats, and fatigue [4]. When symptoms occur, echocardiography should be performed since it is the most useful examination for diagnosing pericardial effusion and detecting signs of tamponade. Although the diagnosis of cardiac tamponade is based on established clinical criteria, an accurate and early diagnosis of tamponade can often be established using echocardiography [5].

When neoplastic cardiac tamponade is diagnosed, drainage by pericardiocentesis should be performed to relieve symptoms and prevent sudden death. A high rate of recurrence $(40-70 \%)$ has been reported due to the persistence of carcinomatous pericarditis-induced pericardial effusion [6]. Therefore, systemic and localized
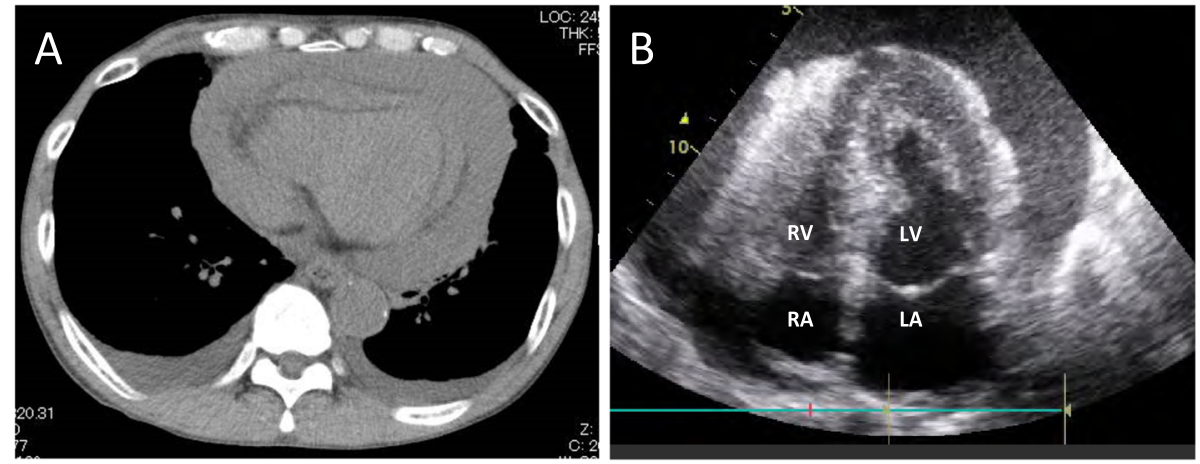

Fig. 1 a Computed tomography findings. Extensive pericardial effusion and bilateral pleural effusion. b Echocardiography (apical four-chamber view) findings. An echo-free space around the heart revealed severe pericardial effusion. $L A$ left atrium, $L V$ left ventricle, $R A$ right atrium, $R V$ right ventricle 

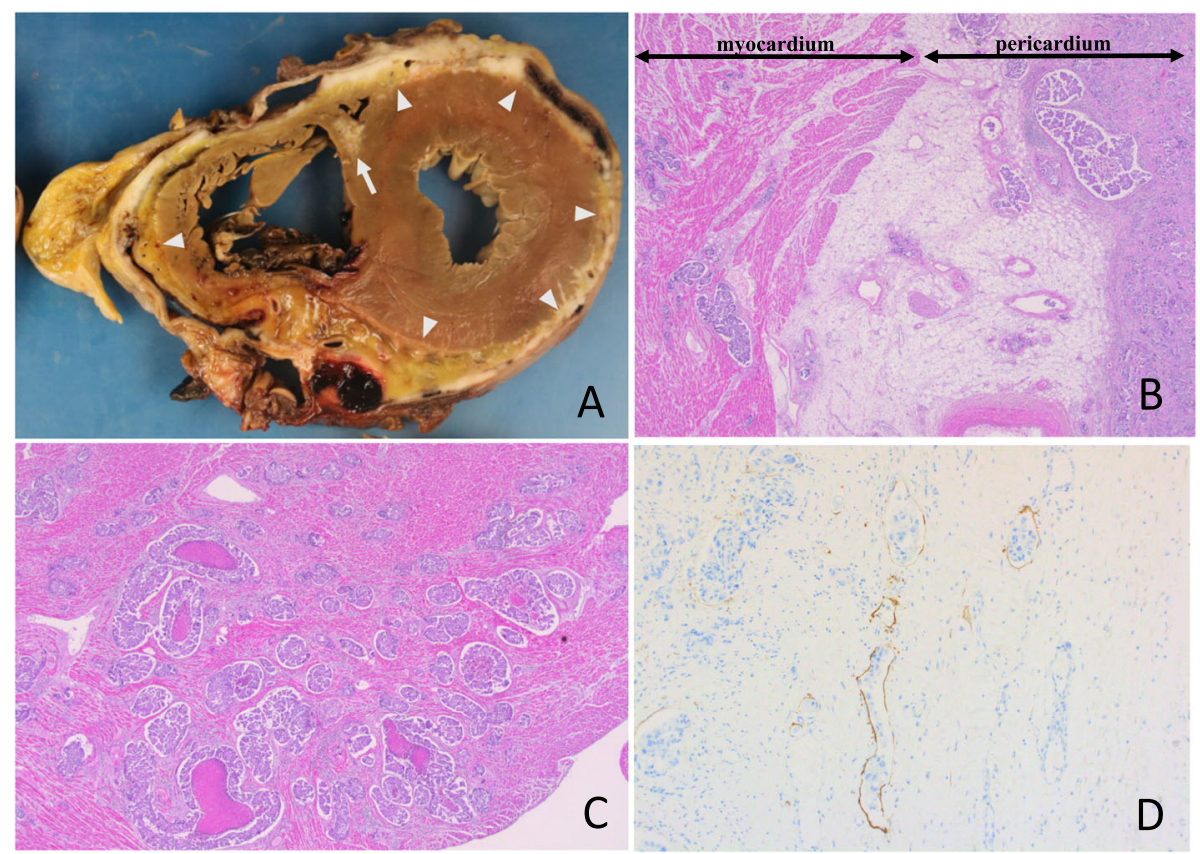

Fig. 2 a Cancerous pericarditis: the parietal pericardium and visceral pericardium adhered to each other (arrow heads) and exhibited tumor infiltration (arrow). b Hematoxylin and eosin-stained pericardial tissue. A large number of tumor cells infiltrated the pericardium. Tumor cells infiltrated the lymph vessels of the myocardium $(\times 20)$. c Hematoxylin and eosin-stained pericardial tissue. A large number of tumor cells infiltrated the myocardium $(\times 20)$. $\mathbf{d}$ Immunostained (D2-40) pericardial tissue. Findings of pericardial lymphatic invasion and lymphatic metastasis $(\times 100)$

treatment should be provided to suppress pericardial effusion. Localized treatment includes percutaneous drainage, anticancer agent injection to destroy pericardial adhesions, and surgical resection of the pericardium. The most appropriate procedure should be selected based on the patient's general condition. Intrapericardial treatment should be tailored to the type of tumor. For example, tris(1-aziridinyl)phosphine sulfide (Thiotepa) is recommended for patients with breast cancer, and cisplatin is recommended for patients with lung cancer [7]. The course after pericardial drainage has been reported to vary according to the type of cancer [4].

Malignant pericardial effusion is associated with an unfavorable prognosis. Wang et al. and Gross et al. reported that the mean survival times after detection of malignant pericardial effusion by pericardiocentesis were 3.1 and 3.7 months, respectively $[8,9]$. To the best of our knowledge, no reports of intrapericardial treatment or endocardial treatment in patients with pancreatic cancer are available. We continued only systemic treatment because intrapericardial treatment could have caused diastolic dysfunction.

Several mechanisms regarding primary tumor metastasis to the pericardial cavity have been proposed, including lymphatic spread, hematogenous spread, systemic spread via nerves, and direct extension. Most tumors metastasize to the heart through the mediastinal lymph nodes. An autopsy was performed in this case, and a main lesion in the head of the pancreas with extensive fibrosis was observed. Moreover, active tumor cells remained. Vessel invasion and nerve invasion were observed, which were considered to have caused the metastasis to other organs. In the heart, the pericardium and myocardium showed strong tumor adhesion, which was considered indicative of cancerous pericarditis. Tumor cell infiltration was observed around almost the entire pericardium, and extensive lymphatic and nerve invasion was observed. Tumor cells also spread to the lymphatic vessels in the heart parenchyma, and some had infiltrated between myocardial fibers. Reports indicate that aminoglycoside antibacterial agents reduced cardiac contractility [10]. In this case, these types of antibiotics were not used, and the occurrence of cardiac dysfunction was considered unlikely. From the pathological results, the infiltration of cancer cells into the myocardium was considered remarkable, and the progression of the cancer caused a decrease in cardiac function, ultimately leading to death. Similar to that in the pericardium, severe lymphatic invasion and venous invasion were observed in several organs. Therefore, the tumor was considered to have reached the pericardium via a lymphatic or hematogenous route where it formed an infiltrating lesion. In the lung, metastasis formation, intravascular tumor embolism, and pleural dissemination were 
observed. The bronchiolar and bronchial walls showed high degrees of tumor invasion, and the peripheral airway was constricted, likely causing respiratory failure. Debilitation occurred throughout the body due to cachexia. Hepatic dysfunction occurred due to tumor infiltration into the liver. Thus, in this case, the cause of death was considered tumor metastasis, including pericardial metastasis, throughout the body. Pericardial metastasis of pancreatic cancer may suggest cancer metastasis throughout the whole body.

\section{Conclusion}

Pericardial metastasis and cardiac tamponade in pancreatic cancer are extremely rare. In this case, lymphatic and vascular invasion caused metastasis to the pericardium and myocardium. In our patient, the interval between tamponade and death was very short (42 days). Pericardial metastasis may be an indication of systemic metastases, and tamponade can be considered an indicator of a poor prognosis. These possibilities are important to consider when pericardial effusion develops in patients with pancreatic cancer.

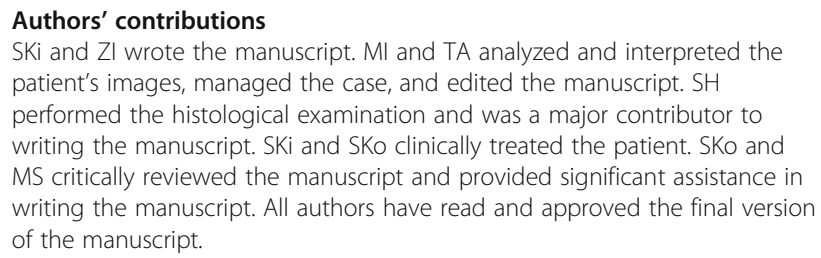

\section{Funding}

The authors did not obtain specific grant funding for this research. The authors declare that they have no competing interests.

\section{Ethical approval and consent to participate}

This study was conducted in accordance with the basic principles of the Declaration of Helsinki.

\section{Consent for publication}

Written informed consent was obtained from the patient's next of kin for publication of this case report and any accompanying images. A copy of the written consent is available for review by the Editor-in-Chief of this journal.

\section{Competing interests}

None declared.

\section{Author details}

'Division of Gastroenterology and Hepatology, Department of Internal Medicine, The Jikei University School of Medicine, Kashiwa Hospital, 163-1 Kashiwa-shita, Kashiwa, Chiba 277-8567, Japan. 'Department of Pathology, The Jikei University School of Medicine, Kashiwa Hospital, Kashiwa City, Chiba 277-8567, Japan. 'Division of Gastroenterology and Hepatology, Department of Internal Medicine, The Jikei University School of Medicine, Tokyo 105-8461, Japan.

Received: 14 August 2020 Accepted: 2 October 2020

Published online: 07 November 2020

\section{References}

1. Vaitkus PT, Herrmann HC, LeWinter MM. Treatment of malignant pericardial effusion. JAMA. 1994;272:59-64.
2. Buck M, Ingle JN, Giuliani ER, Gordon JR, Therneau TM. Pericardial effusion in women with breast cancer. Cancer. 1987;60:263-9.

3. Berge T, Sievers J. Myocardial metastases. A pathological and electrocardiographic study. Br. Heart J. 1968:30:383-90.

4. Takayama T, Okura Y, Okada Y, Honma K, Nashimoto A, Sato N, et al. Characteristics of neoplastic cardiac tamponade and prognosis after pericardiocentesis: a single-center study of 113 consecutive cancer patients. Int J Clin Oncol. 2015;20:872-7.

5. Cheitlin MD, Armstrong WF, Aurigemma GP, Beller GA, Bierman FZ, Davis JL, et al. ACC/AHA/ASE 2003 guideline update for the clinical application of echocardiography--summary article: a report of the American College of Cardiology/American Heart Association Task Force on Practice Guidelines (ACC/AHA/ASE Committee to Update the 1997 Guidelines for the Clinical Application of Echocardiography). J Am Coll Cardiol. 2003;42:954-70.

6. Adler Y, Charron P, Imazio M, Badano L, Baron-Esquivias G, Bogaert J, et al. 2015 ESC Guidelines for the diagnosis and management of pericardial diseases. Kardiol Pol. 2015;73:1028-91.

7. Adler Y, Charron P, Imazio M, Badano L, Baron-Esquivias G, Bogaert J, et al. 2015 ESC Guidelines for the diagnosis and management of pericardial diseases: The Task Force for the Diagnosis and Management of Pericardial Diseases of the European Society of Cardiology (ESC) Endorsed by: The European Association for Cardio-Thoracic Surgery (EACTS). Eur Heart J. 2015; 36:2921-64

8. Wang ML, Liao WB, Bullard MJ, Lin FC, Lin PJ, Chiang CW, et al. Cardiac tamponade in Taiwan. Jpn Circ J. 1997;61:767-71.

9. Gross JL, Younes RN, Deheinzelin D, Diniz AL, Silva RA, Haddad FJ. Surgical management of symptomatic pericardial effusion in patients with solid malignancies. Ann Surg Oncol. 2006;13:1732-8.

10. Adams HR et al. Cardiac toxicities of antibiotics. Environ Health Perspect. 1978;26:217-23.

\section{Publisher's Note}

Springer Nature remains neutral with regard to jurisdictional claims in published maps and institutional affiliations.
Ready to submit your research? Choose BMC and benefit from:
- fast, convenient online submission
- thorough peer review by experienced researchers in your field
- rapid publication on acceptance
- support for research data, including large and complex data types
- gold Open Access which fosters wider collaboration and increased citations
- maximum visibility for your research: over $100 \mathrm{M}$ website views per year
At BMC, research is always in progress.
Learn more biomedcentral.com/submissions 